\title{
A Design Of Monitoring And Diagnosis System Based On WSN For C3I Equipment
}

\author{
Weiqiang $\mathrm{Xu}^{1, \mathrm{a}}$, Guoshun Chen ${ }^{2, \mathrm{~b}}$, Bojian Liü, \\ ${ }^{1}$.School of Ordnance Engineering College, Shijiazhuang 050000, China; \\ ${ }^{2}$.Institute of Ordnance Technology,Shijiazhuang 050000, China \\ ${ }^{3}$.Northwestern Polytechnical University,Xi'an 710000,China \\ aE-mail: 392826314@qq.com
}

Keywords: WSN,C3I System, Monitoring , Fault Diagnosis.

\begin{abstract}
In weapon equipment's diagnosis and maintenance, the current method that operation in the field can not meet actual demand. Remote monitoring and diagnosis was an efficient and practical way to solve the problem. A design of monitoring and diagnosis system for C3I equipment based on Wireless sensor network (WSN) was put forward. In the system, ZigBee was used as the way of communication between wireless nodes. Low power design of WSN was realized by network node design and wireless communication control. The structure design of terminal testing software in man-machine interactive was shown. The system could be used to realize remote monitoring of C3I system working state theoretically on terminal computer and give maintenance suggestion, which could surely enhance the mobility and maintainability of equipment support.
\end{abstract}

\section{Introduction}

In recent years, the traditional monitoring and diagnostic techniques could hardly meet the need of engineering test and measurement due to weapon equipment system is becoming more and more intelligent, complex and integrated. Especially in the promotion process of the army's informatization, the application of electronic technology, computer network technology and other advanced technology in C3I(Command, Control, Communications and Intelligence,C3I) system is increasingly extensive and broad. The characteristic of distribution and network has become increasingly prominent, which leads to more requirements on corresponding monitoring and diagnosis.

With the continuous advancement of computer network and sensor technology, monitoring and diagnosis technology is also advancing, showing a new feature of network and distribution. WSN is a new information acquisition and processing technique. Real-time information can be monitored, sensed and collected from the objective world with WSN[1]. At the same time, the objective world can be also affected through controlling WSN. The emergence of WSN has expanded the interaction between human and nature. It consists of several sensor nodes with a plurality of function, with distribution and self-organizing characteristics, which meet the need of modern testing technology in the aspects of network and distribution.

\section{Application Requirements Analysis Of WSN In The Monitoring And Diagnosis}

As is known to all, C3I system in modern military science is known as the "Force Multiplier" and plays a vital role in modern war. However, there are still some outstanding problems in the monitoring and diagnosis of the system. On the one hand, from the system composition point of view, the complex system consists of the command, control, monitoring, communication, computers, intelligence, surveillance and reconnaissance systems.On the other hand, it has a hierarchical structure which has a subordinate relationship with the military equipment.Each workstation is composed of different working vehicles and equipment [2]. C3I system is featured with diversity and complexity in function and distribution in space.

Generally speaking, some key equipment's failure and damage result in the phenomenon that some nodes cannot be reached, so the system is incapable of normal command and training, 
affecting the generation of fighting force. Despite test and maintenance equipment for electronic equipment will be equipped with the allegations system, but these devices are limited for the C3I system's monitoring and diagnosis capability, which can hardly meet the requirements--quick response to the situation in modern war. Therefore, it is necessary to built a monitoring and test system with distribution and network characteristics to meet the testing requirements of the whole system.

WSN is featured with self organization, distribution, good expansibility and low power consumption. It has a strong ability to adapt to harsh and special environment. It has a great advantage on environmental monitoring and engineering control. With the combination of WSN technology and the corresponding intelligent testing and diagnosis platform, it provides a very convenient way for testing and diagnosis of large-scale complex weapon system (such as C3I system) and health forecast management. Therefore, the information of each subsystem and each C3I equipment could be effectively and timely collected. Also the monitoring and management of the equipment working state will be realized, which largely reduce expenditure of man and material resources. This can meet the demand of C3I system's monitoring and diagnosis in battle field.

\section{Design Of Monitoring And Diagnosis System Based On WSN}

This system uses WSN to realize information collection and simple processing concerning C3I system equipment, and then data is sent to the local server through the way of single hop or multi-hop relay. Local server contacts with remote management terminal through the network and finally human-computer interaction function will be achieved. In the man-machine interactive terminal, it can complete the complex fault intelligent diagnosis and health management function by professional software. The basic framework of the system is shown in Figure 1

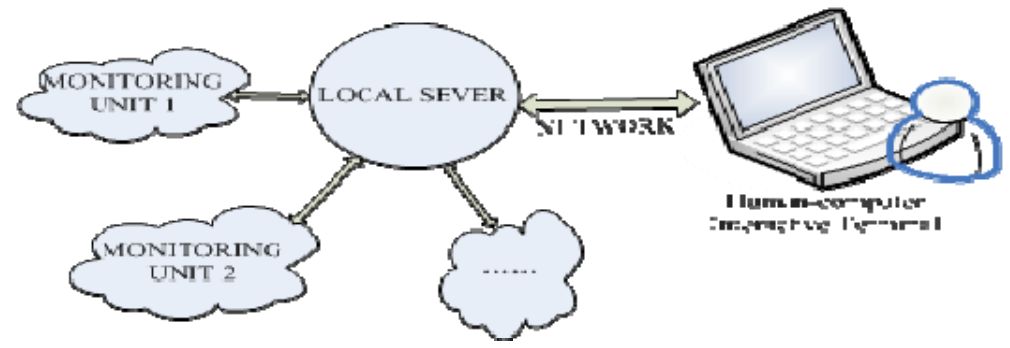

Figure 1: Design Of System's Structure

The monitoring units, located in the bottom and composed of a central processor, a sensor, a GPS module and so on, are mainly distributed in equipment, responsible for acquiring working state parameter of equipment. The data is transported to the local server through customizing protocol, then the local server can analyze these data and also transmit information to a remote terminal, seeking help from expert system. According to various requirements of the system, the data types and the unit are obviously different. The physical quantities are such as voltage, current, frequency, power, electric field intensity and so on. So appropriate sensor type should be selected according to actual testing needs and the corresponding information acquisition task will be completed [3].

Located as a middle bridge is the local server, which role is mainly receiving the monitoring information from monitoring unit and save these data to the database to query and analyze the state of C3I equipment. At the same time, the server transmits data in the database to the Remote Administrator Server in order to make the professional test and measurement in time.

The top part is human-computer interactive terminal, it can effectively realize query and management of the basic monitoring unit through analyzing data received from local server by professional software. According to GPS information or IP address, the positioning could be determined. At the same time, the received information is processed and analyzed by using man-machine interactive terminal system software. So the monitoring and diagnosis of the equipment could be achieved. 


\section{Key Technical Design Analysis}

Low Power Design For Network Node. In order to ensure the reliability of WSN and small volume of wireless sensor node, low power design in WSN is particularly important [4]. In WSN, network nodes are composed of four parts, which is shown in Figure 2. With the rapid development of integrated circuit technology, sensor technology has been very mature and its power consumption is very low.

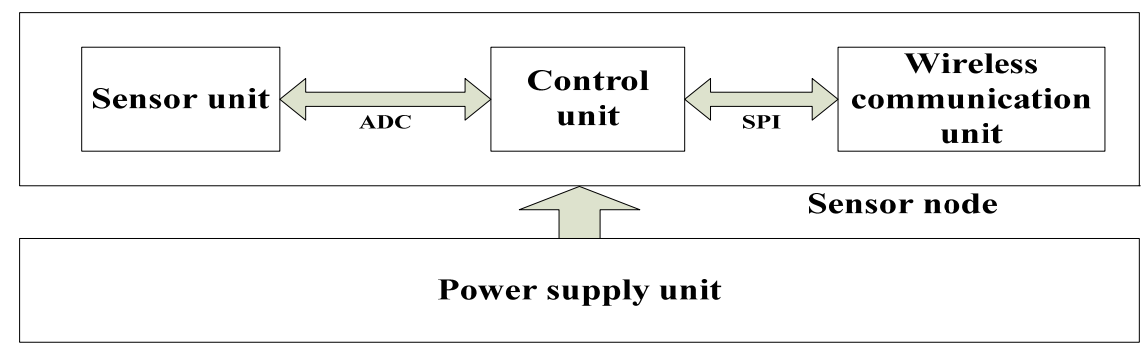

Figure2: Constituent Parts Of WSN

Therefore, the sensing element has little effect on the overall energy consumption of the system. Control unit and wireless communication unit are responsible for most work of the network node, their energy consumption occupies most of the power consumption of the system. So the low power design for WSN is mainly realizing low power design of control unit and wireless communication unit.

Control chip acts as the processor of the network node, its energy consumption is mainly derived from the energy consumption of the instruction. Therefore, the control chip should choose a low operating voltage and low power management capabilities of the processor chip.Most of the energy consumption of the network nodes is due to the wireless communication unit. While the wireless communication unit is mainly responsible for the network communication, and the energy consumption is mainly used for the wireless communication of the network. Therefore, reducing the energy consumption in the process of wireless communication can effectively reduce the power consumption of the wireless communication unit, thus reducing the power consumption of the whole system. Energy consumption can be reduced by the following methods: Reducing the communication flow mode; Reducing data conflict; Reducing the power consumption of wireless communication by means of power control; Using the sleep mode to reduce the power consumption of wireless communication [5].

Data Collection. There are three types of data collection: time driven, event driven, and application driven. In practical applications, it is usually a synthesis of the metioned types. Especially in the C3I system, the testing task is complex due to there are differences among testing requirements. The hybrid type is beneficial to complete the testing tasks of different equipment, saving WSN nodes and improving monitoring efficiency.

Each sensor node of the WSN collects the equipment working status information. The analog signal is converted to digital signal and then uploaded to the local server. The wireless communication distance of sensor nodes is short so multi-hop transmission mode could be used. Sensor node is not only data producer, but also the routing relay nodes, which is quite different from the traditional network[6]. The local server collects useful information from the wireless sensor network and sends the information to the outside world by satellite radio, the Internet or other means. After the analysis and processing, the results will be shown to relevant technical staff. Then they can carry on the real-time monitoring on the equipment, also analyze and deal with the data for research, realizing health management and the fault forecast function. Besides, The local server can submit external feedback to WSN, such as new task, correction of some procedures, etc.

WSN Communication Mode Selection.Wireless Personal Area Network (WPAN) has the following technical standards: HomeRF, Bluetooth, UWB, ZigBee, Wi-Fi technology, etc.. As is shown in Table 1. 
Table1 :Comparision Of Several Common WPAN

\begin{tabular}{llccc}
\hline & Bluetooth & Wi-Fi & Zigbee & HomeRF \\
\hline $\begin{array}{l}\text { Power } \\
\text { Consumption }\end{array}$ & Higher & High & Low & High \\
$\begin{array}{l}\text { Coverage } \\
\text { Transmission }\end{array}$ & $10 \mathrm{~m}$ & $100 \mathrm{~m}$ & $10-75 \mathrm{~m}$ & $50 \mathrm{~m}$ \\
$\begin{array}{l}\text { Rate } \\
\text { Working }\end{array}$ & $1 \mathrm{Mbps}$ & $11 \mathrm{Mbps}$ & $20-250 \mathrm{Kbps}$ & $1.2 \mathrm{Mbps}$ \\
$\begin{array}{l}\text { Frequency } \\
\text { Modulation }\end{array}$ & GFSK & QPSK & QPSK、BPSK & FSK \\
Safety & High & High & High & High \\
\hline
\end{tabular}

There are a lot of communication standards for specific applications in the WPAN field. Bluetooth is generally used in hand-held devices (such as mobile phones, headphones, wireless keyboard, etc.), its power consumption is relatively large and suitable for point to point communication network. Wi-Fi has a slight decrease in power consumption and suits for the formation of local area network in a certain range, such as home, office, etc. HomeRF is suitable for building a smart home and intelligent network. ZigBee's transmission rate is low and not suitable for large-scale data transmission. It can form a network with much functions and suitable for engineering control and environmental monitoring[7]. Thus, ZigBee is the most suitable for communication in the system between nodes.

Terminal Testing Software Design.Because the working mechanism of the equipment and the testing requirements are not the same, the terminal testing software is also different. Modular design is often put to use, including Performance testing subsystem; Achieving equipment's performance; Expert diagnosis subsystem, which includes knowledge acquisition, experience expression, fault database establishment; Maintenance information subsystem, storing instructions maintenance, an album of repair paintings, electrical schematic diagram and so on; Case maintenance subsystem, realizing development and maintenance functions of the expert knowledge base[8]. The main function structure of the testing software is shown in figure 3.

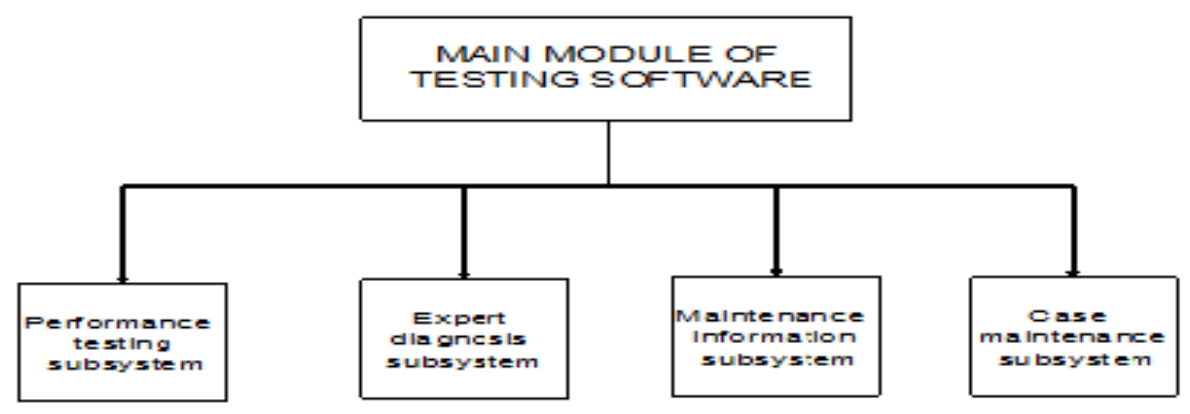

Figure3:Main Module Of Testing Software

\section{Summary}

In combination with the application requirement of the C3I system's testing, the design of the monitoring and diagnosis system based on the WSN for C3I equipment was put forward. The Zigbee technology was used to implement the self-organizing network among the nodes in the system. The functions of data's acquisition and transmission were accomplished. Also, the design of low power for WSN was given. The description of the working principle and key technology of the system were accounted in detail to prove its validity and practicability. The efficiency of C3I system's monitoring and could be obviously improved and the reliability and maintainability of C3I 
system could be enhanced as well. WSN has a promising application prospect in the field of modern weapon equipment's monitoring and diagnosis.

\section{References}

[1] Zhipeng $\mathrm{Hu}$, Et Al. "An Intrusion Detection Algorithm For WSN Based On Kernel Fisher Discriminant." Chinese Journal Of Sensors \& Actuators25.2(2012):246-250.

[2] Yuehua Fan, Bailin Liu, Gong Chaoyang. Design And Implementation Of Fault Diagnosis Expert System Of Command And Control System. Fire Control And Command Control, (2005).30(7), 61-64.

[3] Peng Chen, et al. "A Distributed Testing System of Wireless Sensor Networks." Chinese Journal of Sensors \& Actuators 25.11(2012): 1533-1538.

[4] Qiang Li . Key Technology Research For The Low-Power Node Design Based On Wireless Sensor Networks Master's thesis.Harbin Institute Of Technology, China(2011).

[5] Futao Liu . Research And Design Of Low-Power Wireless Sensor Network For Small-Sale Communication. Master's thesis, Hangzhou Dianzi University. (2013).

[6] Guohua Li. High-Efficiency Data Transmission Methods In Wireless Sensor Networks.Master's thesis.Harbin Institute of Technology(2014).

[7] Wei Yanmei. Design Method Of Wireless Sensor Network Test Platform Master's thesis, Shandong University(2013).

[8] Qingbao Yu, Guoshun Chen, Du Pengfei, \& Wang Peiyuan. Research On Automatic Testing And Diagnosis Equipment For Communication Controller Of Command System, (2010). 33(17), 105-107.

[9] Pfeifera K B, Cemoseka R W.Marline J. An Examination of System Architectures for Distributing Sensor Data Via Ethernet Networks .http ://infoserve. sandia. gov ,2003.

[10]Analog Devices Inc. The ADuC812 MicroConverter as an IEEE 1451. 2 Gompatible Smart Transducer Interface.http://www. analog. com/microconverter,2003. 